\title{
The clinical use of Kampo medicines (traditional Japanese herbal treatments) for controlling cancer patients' symptoms in Japan: a national cross-sectional survey
}

\author{
Satoru Iwase ${ }^{1}$, Takuhiro Yamaguchi ${ }^{2}$, Tempei Miyaji ${ }^{3^{*}}$, Kiyoshi Terawaki ${ }^{4}$, Akio Inui ${ }^{5}$ and Yasuhito Uezono ${ }^{4}$
}

\begin{abstract}
Background: Kampo medicines are traditional Japanese medicines produced from medicinal plants and herbs. Even though the efficacy of Kampo medicines for controlling cancer-related symptoms is being reported, their actual nationwide clinical use has not been comprehensively investigated. We aimed to investigate physicians' recognition of Kampo medicines and their clinical use for cancer patients in the field of palliative care.

Methods: A cross-sectional self-administered anonymous questionnaire was distributed to 549 physicians working in palliative care teams at 388 core cancer treatment hospitals and 161 certified medical institutions that have palliative care units (PCUs).

Results: Valid responses were obtained from 311 physicians (response rate, 56.7\%) who were evenly distributed throughout the country without significant geographical biases. Kampo medicines were prescribed for controlling cancer-related symptoms by $64.3 \%$ of the physicians. The symptoms treated with Kampo medicines were numbness/hypoesthesia ( $n=99,49.5 \%)$, constipation $(n=76,38.0 \%)$, anorexia/weight loss $(n=72,36 \%)$, muscle cramps $(n=71,35.5 \%)$ and languor/fatigue $(n=64,32.0 \%)$. Regarding open issues about prescription, $60.7 \%$ ( $n=173$ ) of the physicians raised the issue that the dosage forms need to be better devised.

Conclusions: To increase the clinical use of Kampo medicines, more evidence from clinical studies is necessary. In addition, their mechanisms of action should be clarified through laboratory studies.
\end{abstract}

Keywords: Kampo, Kampo medicine, Palliative care, Symptom management, Survey

\section{Background}

History of kampo medicine

Kampo medicines are traditional Japanese medicines produced from medicinal plants and herbs. Kampo originates from China and has been adapted to the Japanese culture [1]. Chinese herbal medicine was imported to Japan in $552 \mathrm{AD}$, after which it was uniquely developed into Japanese Kampo [2]. Traditional Chinese Medicine is deeply philosophical and ideological, while Japanese Kampo tends to be more practical and simplified, and relies little on Taoist or other Chinese philosophy [2].

\footnotetext{
* Correspondence: tempeimiyaji@iii.u-tokyo.ac.jp

${ }^{3}$ Interfaculty Initiative in Information Studies, The University of Tokyo, 7-3-1 Hongo, Bunkyo-ku, Tokyo 113-0033, Japan

Full list of author information is available at the end of the article
}

Kampo medicines are currently of great interest to palliative care physicians because of their potential to alleviate the adverse side effects of cancer treatment and improve patients' quality of life.

\section{Use of Kampo and CAM in Japan}

In the past few decades, Kampo has reintegrated into modern medical practice, accompanied by a scientific reevaluation and critical examination of its relevance in conventional medicine [2,3]. Kampo has been used in addition or alternatively to conventional medicines [4]. Currently more than $70 \%$ of Japanese physicians prescribe Kampo medicines in daily clinical practices [5]. Previous survey research has reported that $76 \%$ of the general population in Japan and 50\% of outpatients in 
Tokyo have used some form of CAM and that $10 \%$ of the general population and 19\% of outpatients in Tokyo had used Kampo medicine prescribed by physicians within the last 12 months [6,7]. In addition, the prevalence of use of CAM by cancer patients was $44.6 \%$ in Japan [8]. Internationally, the estimates of CAM use are higher in East Asia and highest in Japan compared to the USA and European countries [9,10]. CAM is often used in palliative care settings where the goal is not cure but rather improvement in QOL [10].

To date, the Ministry of Health, Labour and Welfare (MHLW) has approved the use of 148 Kampo medicines, and the prescription of Kampo medicines is within the national health insurance system [3,11]. Although Kampo can be seen as orthodox from a historical Japanese perspective, it tends to be classified as Complementary and Alternative Medicine (CAM) according to Western conventions. The main reason for this is the lack of scientific evidence of its efficacy and the limited knowledge and spread of this therapy in other regions, especially outside of East Asia.

However, clinical studies of Kampo have been conducted in Japan, and its efficacy has been reported in research papers. For example, a randomized control trial demonstrated that the Kampo medicine Rikkunshito exerted greater effects in alleviating gastrointestinal symptoms than cisapride (a gastroprokinetic agent) [12]. The efficacy of Rikkunshito against non-ulcer dyspepsia (NUD) $[13,14]$, gastrointestinal symptoms after gastrectomy (surgical NUD) [15], functional dyspepsia [16,17], and nausea and vomiting caused by selective serotonin reuptake inhibitors [18] has also been reported. Also, the Japanese Society for Oriental Medicine has compiled comprehensive data on randomized controlled trials of Kampo medicine in Japan, published as "Evidence Reports of Kampo Treatment" (EKAT) [19]. In addition to clinical trials, the potential mechanisms of action of Kampo medicines are also starting to be reported [20].

As described above, there is increasing evidence of the efficacy of Kampo medicines and increasing attention has been given to their clinical application. However, there has been no comprehensive investigation of the use of Kampo medicines in cancer treatment. Therefore, we conducted a nationwide survey of the current use of Kampo medicines for cancer-related treatment and of physicians' attitudes toward using Kampo medicines in Japan.

\section{Methods}

\section{Study sample and data collection}

The survey was carried out between January and March of 2011, by mailing a self-administered anonymous questionnaire to 549 palliative care physicians who administer chemotherapy to cancer patients or who are involved in their terminal care. The palliative care teams in 388 core cancer treatment hospitals and 161 palliative care units (PCUs) within medical institutions were selected because they represent palliative care practice in Japan. This included all core cancer treatment hospitals and PCUs in Japan as of February 2011. Core cancer treatment hospitals are the medical facilities specified by the MHLW to provide high-quality expert care for cancer patients. These facilities are established within each prefecture in Japan, according to the principles set forth in the Cancer Control Act promulgated in April 2007. The contact information of subjects was obtained from a web site of the Cancer Control Information Center, National Cancer Center [21].

We did not specifically include general internists or surgeons who are not in charge of palliative care as subjects of the survey. This is because the certification system for the palliative care specialist is still immature in Japan and the attending physicians of palliative care teams and PCUs are often internists or surgeons.

\section{Questionnaire development}

An eight-page, 18-item questionnaire was designed in Japanese. It covered four categories: (1) status of cancer treatment and use of Kampo medicines, (2) cancer cachexia and utilization of Kampo medicines (data not shown), (3) adverse side effects of anti-cancer drugs and utilization of Kampo medicines, and (4) background variables. Although the questionnaire was not formally validated, the questionnaire and its items were designed and formulated based upon the expert opinions of specialists from palliative care, medical oncology, Kampo medicine, and biological statistics, and also from literature reviews. It was finalized after testing several samples.

\section{Ethical considerations}

We conducted this research in compliance with the Helsinki Declaration. We had requested an ethical review of this research from the ethical review committee of the National Cancer Center prior to commencement. However, since this research involves neither patients' data nor intervention, the committee judged that this research should not be subjected to any Japanese medical research guidelines. Accordingly, the research was exempt from the requirement for formal ethical approval.

To ensure that informed consent was obtained, the questionnaire was sent to the physicians with a leaflet explaining the survey's objectives and that (1) each subject was free to decide whether or not to answer the questions; (2) the collected data will be processed and analyzed anonymously; and (3) the data will be securely archived by the Research Secretariat. Consent was implied through the return of a completed questionnaire. 


\section{Data analysis}

The collected data were entered into an electronic database and analyzed using SPSS (IBM, New York, USA). Chi-squared tests ( $p$ value $<0.050$ ) were conducted to compare the frequency distributions of two cross-tabulations. The first was physicians in the palliative care teams at the core cancer treatment hospitals compared with physicians in the PCUs. The second was the palliative care specialists certified by the Japan Society of Palliative Medicine (JSPM) compared with non-specialists.

\section{Results and discussion}

Of the 549 questionnaires distributed, 311 valid responses were collected for analysis (response rate, 56.7\%). Responses were obtained from 226 physicians (response rate, $58.2 \%$ ) at core cancer treatment hospitals (palliative care team physicians) and 79 physicians (response rate, 49.1\%) from PCUs (PCU physicians). With the moderate rate of valid responses $(56.7 \%)$, the respondents were well-distributed throughout the country, without significant geographical biases. Table 1 shows the response rates and the respondents' background characteristics. Two hundred thirty seven respondents (77.9\%) were aged between 40 and 59 years. Two hundred seventy three respondents (90.1\%) were male, and 128 respondents (41.2\%) were JSPM-authorized palliative care specialists (including provisional medical advisors).

\section{Difficult to treat cancer-related symptoms}

Physicians were asked to identify which of the 23 common cancer-related symptoms that they find difficult to treat (Table 2). More than $50 \%$ of the physicians identified numbness/hypoesthesia ( $\mathrm{n}=240,77.2 \%)$, languor/fatigue ( $\mathrm{n}=225,72.3 \%)$, delirium $(\mathrm{N}=170,54.7 \%)$, and taste alteration ( $\mathrm{n}=166,53.4 \%)$. In comparison with the PCU physicians, more palliative care team physicians identified taste alteration ( $\mathrm{p}=0.029)$, nausea/vomiting (during chemotherapy) $(\mathrm{p}=0.000)$, and constipation (caused by opioid use) $(\mathrm{p}=0.038)$. More of the PCU physicians, on the other hand, reported having difficulty treating adjustment disorder $(\mathrm{p}=0.014)$. In addition, the symptoms of taste alteration $(\mathrm{p}=0.050)$, dysphagia/deglutition disorder $(\mathrm{p}=0.036)$ and muscle weakness $(\mathrm{p}=0.047)$ were

Table 1 Respondents' background characteristics

\begin{tabular}{|c|c|c|c|c|c|c|}
\hline Respondents $(n=311)$ & & Average \pm SD & $\begin{array}{l}\text { Minimum } \\
\text { value }\end{array}$ & $\begin{array}{l}\text { Maximum } \\
\text { value }\end{array}$ & & \\
\hline Age & & $49 \pm 8$ & 28 & 75 & & \\
\hline Years of experience & & $23 \pm 8$ & 4 & 50 & & \\
\hline & & Responses & $\%$ & & & \\
\hline Institution $(n=549) *$ & & & & & & \\
\hline Core cancer treatment hospital $(n=388)$ & & 226 & 58.2 & & & \\
\hline Palliative Care Unit in medical institution $(n=161)$ & & 79 & 49.1 & & & \\
\hline & & $n$ & $\%$ & & & \\
\hline Age group & & & & & & \\
\hline $20-29$ years & & 1 & 0.3 & & & \\
\hline $30-39$ years & & 39 & 12.8 & & & \\
\hline 40-49 years & & 119 & 39.1 & & & \\
\hline $50-59$ years & & 118 & 38.8 & & & \\
\hline$\geq 60$ years & & 27 & 8.9 & & & \\
\hline Sex & & & & & & \\
\hline Male & & 273 & 90.1 & & & \\
\hline Female & & 30 & 9.9 & & & \\
\hline Palliative Care Specialists certified by JSPM ${ }^{* *}$ & & & & & & \\
\hline Specialists (including provisional medical advisors) & & 128 & 41.2 & & & \\
\hline Non-specialists & & 183 & 58.8 & & & \\
\hline Hokkaido-Tohoku & Kanto & Chubu & Kinki & Chugoku & Shikoku & Kyushu-Okinawa \\
\hline Number of questionnaires distributed & 116 & 92 & 91 & 47 & 27 & 97 \\
\hline Number of responses & 43 & 41 & 33 & 25 & 11 & 37 \\
\hline Response rate (\%) & 37.1 & 44.6 & 36.3 & 53.2 & 40.7 & 38.1 \\
\hline
\end{tabular}

*Six responses had missing institution data, and ***95 responses had missing region data.

** JSPM: Japan Society for Palliative Medicine. 
Table 2 Difficult to treat cancer-related symptoms identified by physicians

\begin{tabular}{|c|c|c|c|c|c|c|c|c|c|c|c|c|}
\hline \multirow[t]{2}{*}{ Symptoms } & \multicolumn{2}{|c|}{$\begin{array}{c}\text { All physicians } \\
(\mathrm{n}=311)\end{array}$} & \multicolumn{2}{|c|}{$\begin{array}{l}\text { Palliative care } \\
\text { teams }(n=226)\end{array}$} & \multicolumn{2}{|c|}{$\begin{array}{c}\text { PCUs } \\
(n=79)\end{array}$} & \multirow[t]{2}{*}{ p-value } & \multicolumn{2}{|c|}{$\begin{array}{l}\text { Specialists } \\
(\mathrm{n}=128)\end{array}$} & \multicolumn{2}{|c|}{$\begin{array}{l}\text { Non-specialists } \\
(n=183)\end{array}$} & \multirow[t]{2}{*}{ p-value } \\
\hline & frequency & $\%$ & frequency & $\%$ & frequency & $\%$ & & frequency & $\%$ & frequency & $\%$ & \\
\hline Numbness/Hypesthesia & 240 & 77.2 & 180 & 79.6 & 55 & 69.6 & 0.165 & 99 & 77.3 & 141 & 77.0 & 1.000 \\
\hline Languor/Fatigue & 225 & 72.3 & 161 & 71.2 & 61 & 77.2 & 0.276 & 99 & 77.3 & 126 & 68.9 & 0.122 \\
\hline Delirium & 170 & 54.7 & 119 & 52.7 & 48 & 60.8 & 0.447 & 73 & 57.0 & 97 & 53.0 & 0.490 \\
\hline Taste alteration & 166 & 53.4 & 124 & 54.9 & 42 & 53.2 & 0.029 & 77 & 60.2 & 89 & 48.6 & 0.050 \\
\hline Edema (Local edema/Anasarca) & 150 & 48.2 & 109 & 48.2 & 39 & 49.4 & 0.821 & 59 & 46.1 & 91 & 49.7 & 0.565 \\
\hline Pain & 146 & 46.9 & 113 & 50.0 & 31 & 39.2 & 0.226 & 55 & 43.0 & 91 & 49.7 & 0.250 \\
\hline Anorexia/Weight loss & 140 & 45.0 & 109 & 48.2 & 30 & 38.0 & 0.108 & 64 & 50.0 & 76 & 41.5 & 0.165 \\
\hline Abdominal discomfort & 131 & 42.1 & 98 & 43.4 & 31 & 39.2 & 0.735 & 55 & 43.0 & 76 & 41.5 & 0.816 \\
\hline Stomatitis/Xerostomia & 122 & 39.2 & 89 & 39.4 & 33 & 41.8 & 0.141 & 54 & 42.2 & 68 & 37.2 & 0.409 \\
\hline Depression & 116 & 37.3 & 86 & 38.1 & 30 & 38.0 & 0.175 & 41 & 32.0 & 75 & 41.0 & 0.122 \\
\hline Adjustment disorder & 113 & 36.3 & 73 & 32.3 & 39 & 49.4 & 0.014 & 47 & 36.7 & 66 & 36.1 & 1.000 \\
\hline Dyspnea/Breathlessness & 113 & 36.3 & 77 & 34.1 & 35 & 44.3 & 0.162 & 48 & 37.5 & 65 & 35.5 & 0.811 \\
\hline Nausea/Nomiting (other) & 101 & 32.5 & 75 & 33.2 & 24 & 30.4 & 0.893 & 38 & 29.7 & 63 & 34.4 & 0.392 \\
\hline Dysphagia/Deglutition disorder & 100 & 32.2 & 68 & 30.1 & 31 & 39.2 & 0.281 & 50 & 39.1 & 50 & 27.3 & 0.036 \\
\hline Sleep disorder/Insomnia & 93 & 29.9 & 69 & 30.5 & 23 & 29.1 & 0.796 & 42 & 32.8 & 51 & 27.9 & 0.379 \\
\hline Constipation (caused by opioid use) & 84 & 27.0 & 69 & 30.5 & 15 & 19.0 & 0.038 & 34 & 26.6 & 50 & 27.3 & 0.898 \\
\hline $\begin{array}{l}\text { Nausea/Nomiting } \\
\text { (during chemotherapy) }\end{array}$ & 76 & 24.4 & 71 & 31.4 & 5 & 6.3 & 0.000 & 27 & 21.1 & 49 & 26.8 & 0.284 \\
\hline Muscle weakness & 65 & 20.9 & 46 & 20.4 & 19 & 24.1 & 0.346 & 34 & 26.6 & 31 & 16.9 & 0.047 \\
\hline $\begin{array}{l}\text { Nausea/Nomiting } \\
\text { (caused by opioid use) }\end{array}$ & 61 & 19.6 & 51 & 22.6 & 10 & 12.7 & 0.690 & 24 & 18.8 & 37 & 20.2 & 0.774 \\
\hline $\begin{array}{l}\text { Constipation } \\
\text { (not caused by opioid use) }\end{array}$ & 59 & 19.0 & 47 & 20.8 & 11 & 13.9 & 0.377 & 28 & 21.9 & 31 & 16.9 & 0.305 \\
\hline Muscle cramp & 42 & 13.5 & 31 & 13.7 & 11 & 13.9 & 0.741 & 23 & 18.0 & 19 & 10.4 & 0.064 \\
\hline Diarrhea & 40 & 12.9 & 34 & 15.0 & 6 & 7.6 & 0.136 & 16 & 12.5 & 24 & 13.1 & 1.000 \\
\hline Anemia & 29 & 9.3 & 24 & 10.6 & 5 & 6.3 & 0.344 & 16 & 12.5 & 13 & 7.1 & 0.177 \\
\hline Others & 11 & 3.5 & 6 & 2.7 & 5 & 6.3 & 0.325 & 4 & 3.1 & 7 & 3.8 & 0.770 \\
\hline
\end{tabular}

Multiple answers allowed, p-value based on Chi-square test.

identified as being difficult to treat more often by the palliative care specialists than the non-specialists.

Numbness is a neuropathic symptom that frequently occurs as an adverse side effect of chemotherapy. It has been reported to account for $58 \%$ of all neurological symptoms experienced by cancer patients [22]. Fatigue is the most common cancer symptom [23], and was reported by $66 \%$ of patients in a previous study [22]. The prevalence of delirium is $25-40 \%$ ( $85-88 \%$ in the terminal stage of cancer) [24-26], and the prevalence of taste alteration is 36-75\% among patients receiving chemotherapy [27]. Thus, it was shown in the present survey that the symptoms palliative care physicians have difficulty managing in Japan are those frequently seen in cancer patients.

We also found that the palliative care team physicians confront taste alteration $(\mathrm{p}=0.029)$, nausea/vomiting during chemotherapy $(\mathrm{p}=0.000)$ and constipation during opioid use (0.038) more often than the PCU physicians (Table 2). These facts suggest that the palliative care teams are often in charge of patients receiving chemotherapy, while PCUs are more frequently dealing with psychiatric symptoms than the adverse side effects of chemotherapy.

\section{Prescription of Kampo medicines}

Kampo medicines were being prescribed by $64.3 \%(n=200)$ of the physicians to alleviate the cancer patients' symptoms. Kampo medicines were prescribed to control numbness/ hypoesthesia ( $\mathrm{n}=99,49.5 \%$ ), constipation (not caused by opioid use) ( $\mathrm{n}=76,38 \%)$, anorexia/weight loss $(\mathrm{n}=72,36 \%)$, muscle cramps ( $\mathrm{n}=71,35.5 \%)$, and languor/fatigue $(\mathrm{n}=64$, $32 \%$ ) by more than $30 \%$ of the physicians (Table 3 ). The palliative care team physicians prescribed Kampo medicines for numbness/hypoesthesia $(\mathrm{p}=0.000)$, anorexia/weight loss $(\mathrm{p}=0.046)$, pain $(\mathrm{p}=0.020)$, and nausea/vomiting during chemotherapy $(\mathrm{p}=0.016)$, more frequently than the PCU physicians. This difference may arise because the palliative care teams more often examine patients who are under chemotherapy than the PCUs, and thus they pay more 
Table 3 Symptoms for which Kampo medicines were prescribed

\begin{tabular}{|c|c|c|c|c|c|c|c|}
\hline \multirow[t]{2}{*}{ Symptoms } & \multicolumn{2}{|c|}{$\begin{array}{l}\text { All physicians } \\
(\mathrm{n}=\mathbf{2 0 0})\end{array}$} & \multicolumn{2}{|c|}{$\begin{array}{l}\text { Palliative care teams } \\
(n=149)\end{array}$} & \multicolumn{2}{|c|}{$\begin{array}{c}\text { PCUs } \\
(n=46)\end{array}$} & \multirow[t]{2}{*}{ p-value } \\
\hline & frequency & $\%$ & frequency & $\%$ & frequency & $\%$ & \\
\hline Numbness/Hypesthesia & 99 & 49.5 & 86 & 57.7 & 12 & 26.1 & 0.000 \\
\hline Constipation (not caused by opioid use) & 76 & 38 & 56 & 37.6 & 20 & 43.5 & 0.182 \\
\hline Anorexia/Weight loss & 72 & 36 & 60 & 40.3 & 12 & 26.1 & 0.046 \\
\hline Muscle cramp & 71 & 35.5 & 54 & 36.2 & 17 & 37.0 & 0.279 \\
\hline Languor/Fatigue & 64 & 32 & 49 & 32.9 & 14 & 30.4 & 0.818 \\
\hline Constipation (caused by opioid use) & 48 & 24 & 37 & 24.8 & 11 & 23.9 & 0.490 \\
\hline Abdominal discomfort & 46 & 23 & 29 & 19.5 & 16 & 34.8 & 0.088 \\
\hline Diarrhea & 45 & 22.5 & 39 & 26.2 & 5 & 10.9 & 0.090 \\
\hline Delirium & 40 & 20 & 27 & 18.1 & 13 & 28.3 & 0.155 \\
\hline Pain & 38 & 19 & 35 & 23.5 & 3 & 6.5 & 0.020 \\
\hline Edema (Local edema/Anasarca) & 31 & 15.5 & 25 & 16.8 & 6 & 13.0 & 0.546 \\
\hline Nausea/Nomiting (other) & 27 & 13.5 & 22 & 14.8 & 5 & 10.9 & 0.566 \\
\hline Nausea/Nomiting (during chemotherapy) & 22 & 11 & 22 & 14.8 & 0 & 0.0 & 0.016 \\
\hline Stomatitis/Xerostomia & 21 & 10.5 & 19 & 12.8 & 2 & 4.3 & 0.216 \\
\hline Taste alteration & 20 & 10 & 17 & 11.4 & 3 & 6.5 & 0.409 \\
\hline Depression & 20 & 10 & 17 & 11.4 & 3 & 6.5 & 0.409 \\
\hline Nausea/Nomiting (caused by opioid use) & 17 & 8.5 & 16 & 10.7 & 1 & 2.2 & 0.129 \\
\hline Adjustment disorder & 15 & 7.5 & 12 & 8.1 & 3 & 6.5 & 0.846 \\
\hline Sleep disorder/Insomnia & 14 & 7 & 10 & 6.7 & 4 & 8.7 & 0.823 \\
\hline Others & 13 & 6.5 & 6 & 4.0 & 6 & 13.0 & 0.055 \\
\hline Anemia & 11 & 5.5 & 9 & 6.0 & 2 & 4.3 & 0.805 \\
\hline Dysphagia/Deglutition disorder & 10 & 5 & 9 & 6.0 & 1 & 2.2 & 0.581 \\
\hline Dyspnea/Breathlessness & 6 & 3 & 5 & 3.4 & 1 & 2.2 & 1.000 \\
\hline Muscle weakness & 3 & 1.5 & 3 & 2.0 & 0 & 0.0 & 0.614 \\
\hline
\end{tabular}

Multiple answers allowed, $p$-value based on Chai-square test.

attention than the PCUs to the necessity of controlling the adverse side effects of chemotherapy. Also, PCU patients have more difficulty taking Kampo medicines than the general hospital patients under the palliative care teams. The frequency of prescribing Kampo medicines did not vary significantly across the symptoms between the palliative care specialists and non-specialists.

\section{Reasons for prescription}

More than $60 \%$ of the physicians prescribed Kampo medicines for the following reasons: 'the drug therapy options are greater' $(\mathrm{n}=144,72 \%)$, 'ineffectiveness of other treatments' ( $n=129,64.5 \%)$, and 'unavailability of other appropriate treatments' $(\mathrm{n}=127,63.5 \%)$. Although 'patient demand' was the least frequent reason $(n=46,23 \%)$, palliative care specialists were more attentive to patients' demands than non-specialists $(\mathrm{n}=28,37.3 \%, \mathrm{p}=0.000)$.

\section{Variety and frequency of prescriptions}

Eight Kampo medicines were selected from the literature reviews to investigate frequency of prescription. Table 4 shows the composition of each Kampo medicine [28-30].
Daikenchuto was the most frequently prescribed ( $\mathrm{n}=140$, $70 \%$ ) among eight major Kampo medicines (Table 5). This is probably because the efficacy of Daikenchuto for the treatment of gastrointestinal symptoms is currently being tested in clinical trials in Japan and the United States. A tolerability and efficacy phase II study of Daikenchuto for the treatment of postoperative ileus has been already completed in the United States [31]. This might encourage its prescription by physicians. The palliative care team physicans prescribed Goshajinkigan $(\mathrm{p}=0.000)$, Rikkunshito $(\mathrm{p}=0.001)$, Hochuekkito $(\mathrm{p}=0.011)$, Juzentaihoto $(\mathrm{p}=0.001)$, and Hangeshashinto $(\mathrm{p}=0.000)$ more frequently than $\mathrm{PCU}$ physicians, while there were no significant differences in the medicines prescribed between the palliative care specialists and non-specialists.

\section{Physician-recognized effectiveness}

We investigated the physician-recognized effectiveness of eight Kampo medicines. Two symptoms from each Kampo medicine's package insert were listed and the physicians were asked to indicate whether they believed the medicine effectively treated them (Table 6). More than $50 \%$ of the 
Table 4 Composition of Kampo medicines

Kampo Medicine Ingredients (crude drugs)

\begin{tabular}{|c|c|c|c|c|c|c|c|c|c|c|}
\hline Hangeshashinto & Pinelliae Tuber & $\begin{array}{l}\text { Scutellariae } \\
\text { Radix }\end{array}$ & $\begin{array}{l}\text { Zingiberis } \\
\text { Processum Rhizoma }\end{array}$ & $\begin{array}{l}\text { Glycyrrhizae } \\
\text { Radix }\end{array}$ & $\begin{array}{l}\text { Zizyphi } \\
\text { Fructus }\end{array}$ & $\begin{array}{l}\text { Ginseng } \\
\text { Radix }\end{array}$ & $\begin{array}{l}\text { Coptidis } \\
\text { Rhizoma }\end{array}$ & & & \\
\hline Hochuekkito & Astragali Radix & $\begin{array}{l}\text { Atractylodis } \\
\text { lanceae Rhizoma }\end{array}$ & $\begin{array}{l}\text { Ginseng } \\
\text { Radix }\end{array}$ & $\begin{array}{l}\text { Angelicae } \\
\text { Radix }\end{array}$ & $\begin{array}{l}\text { Bupleuri } \\
\text { Radix }\end{array}$ & $\begin{array}{l}\text { Zizyphi } \\
\text { Fructus }\end{array}$ & $\begin{array}{l}\text { Aurantii Nobilis } \\
\text { Pericarpium }\end{array}$ & $\begin{array}{l}\text { Glycyrrhizae } \\
\text { Radix }\end{array}$ & $\begin{array}{l}\text { Cimicifugae } \\
\text { Rhizoma }\end{array}$ & $\begin{array}{l}\text { Zingiberis } \\
\text { Rhizoma }\end{array}$ \\
\hline Rikkunshito & $\begin{array}{l}\text { Atractylodis } \\
\text { lanceae Rhizoma }\end{array}$ & Ginseng Radix & $\begin{array}{l}\text { Pinelliae } \\
\text { Tuber }\end{array}$ & Poria & $\begin{array}{l}\text { Zizyphi } \\
\text { Fructus }\end{array}$ & $\begin{array}{l}\text { Aurantii Nobilis } \\
\text { Pericarpium }\end{array}$ & $\begin{array}{l}\text { Glycyrrhizae } \\
\text { Radix }\end{array}$ & $\begin{array}{l}\text { Zingiberis } \\
\text { Rhizoma }\end{array}$ & & \\
\hline Juzentaihoto & $\begin{array}{l}\text { Astragali } \\
\text { Radix }\end{array}$ & $\begin{array}{l}\text { Cinnamomi } \\
\text { Cortex }\end{array}$ & $\begin{array}{l}\text { Rehmanniae } \\
\text { Radix }\end{array}$ & Paeoniae Radix & $\begin{array}{l}\text { Cnidii } \\
\text { Rhizoma } \\
\end{array}$ & $\begin{array}{l}\text { Atractylodis } \\
\text { lanceae Rhizoma }\end{array}$ & $\begin{array}{l}\text { Angelicae } \\
\text { Radix }\end{array}$ & Ginseng Radix & Poria & $\begin{array}{l}\text { Glycyrrhizae } \\
\text { Radix } \\
\end{array}$ \\
\hline Yokukansan & $\begin{array}{l}\text { Atractylodis } \\
\text { lanceae Rhizoma }\end{array}$ & Poria & $\begin{array}{l}\text { Cnidii } \\
\text { Rhizoma }\end{array}$ & $\begin{array}{l}\text { Uncariae Uncis } \\
\text { cum Ramulus }\end{array}$ & $\begin{array}{l}\text { Angelicae } \\
\text { Radix }\end{array}$ & Bupleuri Radix & $\begin{array}{l}\text { Glycyrrhizae } \\
\text { Radix }\end{array}$ & & & \\
\hline Shakuyakukanzoto & $\begin{array}{l}\text { Glycyrrhizae } \\
\text { Radix }\end{array}$ & Paeoniae Radix & & & & & & & & \\
\hline Daikenchuto & $\begin{array}{l}\text { Zingiberis } \\
\text { Processum Rhizoma }\end{array}$ & Ginseng Radix & $\begin{array}{l}\text { Zanthoxyli } \\
\text { Fructus }\end{array}$ & & & & & & & \\
\hline Goshajinkigan & $\begin{array}{l}\text { Rehmanniae } \\
\text { Radix }\end{array}$ & $\begin{array}{l}\text { Achyranthis } \\
\text { Radix }\end{array}$ & $\begin{array}{l}\text { Corni } \\
\text { Fructus }\end{array}$ & $\begin{array}{l}\text { Dioscoreae } \\
\text { Rhizoma }\end{array}$ & $\begin{array}{l}\text { Plantaginis } \\
\text { Semen }\end{array}$ & $\begin{array}{l}\text { Alismatis } \\
\text { Rhizoma }\end{array}$ & Poria & $\begin{array}{l}\text { Moutan } \\
\text { Cortex }\end{array}$ & $\begin{array}{l}\text { Cinnamomi } \\
\text { Cortex }\end{array}$ & $\begin{array}{l}\text { Processi } \\
\text { Aconiti Radix }\end{array}$ \\
\hline
\end{tabular}

Ingredients of each Kampo medicine were based on the package inserts of Tsumura products [28].

Scientific names of ingredients were based on Metabolomics.jp [29] and The Japanese Pharmacopeia Fifteenth edition [30]. 
Table 5 The Kampo medicines prescribed by the physicians

\begin{tabular}{|c|c|c|c|c|c|c|c|}
\hline \multirow[t]{2}{*}{ Kampo medicine } & \multicolumn{2}{|c|}{$\begin{array}{l}\text { All physicians } \\
(\mathrm{n}=\mathbf{2 0 0})\end{array}$} & \multicolumn{2}{|c|}{$\begin{array}{l}\text { Palliative care teams } \\
(n=149)\end{array}$} & \multicolumn{2}{|c|}{$\begin{array}{c}\text { PCUs } \\
(n=46)\end{array}$} & \multirow[t]{2}{*}{ p-value } \\
\hline & frequency & $\%$ & frequency & $\%$ & frequency & $\%$ & \\
\hline Daikenchuto & 140 & 70.0 & 109 & 73.2 & 29 & 63.0 & 0.124 \\
\hline Goshajinkigan & 100 & 50.0 & 89 & 59.7 & 11 & 23.9 & 0.000 \\
\hline Rikkunshito & 97 & 48.5 & 82 & 55.0 & 15 & 32.6 & 0.001 \\
\hline Shakuyakukanzoto & 96 & 48.0 & 76 & 51.0 & 20 & 43.5 & 0.069 \\
\hline Hochuekkito & 90 & 45.0 & 76 & 51.0 & 13 & 28.3 & 0.011 \\
\hline Juzentaihoto & 84 & 42.0 & 73 & 49.0 & 11 & 23.9 & 0.001 \\
\hline Yokukansan & 61 & 30.5 & 45 & 30.2 & 16 & 34.8 & 0.253 \\
\hline Hangeshashinto & 54 & 27.0 & 51 & 34.2 & 3 & 6.5 & 0.000 \\
\hline Others & 24 & 12.0 & 20 & 13.4 & 4 & 8.7 & 0.457 \\
\hline
\end{tabular}

Multiple answers allowed, $\mathrm{p}$-value based on Chi-square test.

physicians recognized the effectiveness of Hangeshashinto against diarrhea caused by chemotherapy $(\mathrm{n}=31,53.4 \%)$, of Hochuekkito and Juzentaihoto against fatigue $(\mathrm{n}=54,56.3 \%$ and $\mathrm{n}=50,56.8 \%$ respectively), of Rikkunshito against anorexia $(\mathrm{n}=46,50 \%)$, of Yokukansan against delirium $(\mathrm{n}=38,63.3 \%)$, of Shakuyakukanzoto against leg cramps $(\mathrm{n}=79,82.3 \%)$, and of Daikenchuto against ileus ( $\mathrm{n}=101$, 78.9\%) and opioid-caused constipation and abdominal pain $(\mathrm{n}=62,53.9 \%)$. There was no significant difference in the medicines recognized as effective between the palliative care team and PCU physicians, while the palliative care specialists seemed to be more aware of the effectiveness of Rikkunshito against nausea than non-specialists $(\mathrm{p}=0.012)$
(Table 6). These results suggest that there is consensus among palliative care physicians regarding the effectiveness of particular Kampo medicines against particular symptoms.

\section{Prescription considerations}

In the questionnaire, the physicians were asked, "What are the important considerations when selecting a Kampo medicine for prescription?". More than $80 \%$ of the physicians recognized the importance of 'symptomalleviating effects (alleviation of adverse side effects) ( $n=173,93 \%)$,'alleviation of symptoms that reduce QOL in the terminal stage of cancer' ( $n=162,87.6 \%)$, 'low incidence of adverse side effects' ( $\mathrm{n}=157,84.9 \%)$ and 'easy

Table 6 Physician-recognized effectiveness of Kampo medicines

\begin{tabular}{|c|c|c|c|c|c|c|c|c|}
\hline \multirow[t]{3}{*}{ Kampo medicine } & \multirow[t]{3}{*}{ Symptoms } & \multicolumn{7}{|c|}{ Recognized as effective } \\
\hline & & \multicolumn{2}{|c|}{ All physicians } & \multicolumn{2}{|l|}{ Specialists } & \multicolumn{2}{|c|}{ Non-specialists } & \multirow[t]{2}{*}{ p-value } \\
\hline & & frequency/total & $\%$ & frequency/total & $\%$ & frequency/total & $\%$ & \\
\hline \multirow[t]{2}{*}{ Hangeshashinto } & Diarrhea caused by chemotherapy & $31 / 58$ & 53.4 & $10 / 22$ & 45.5 & $21 / 36$ & 58.3 & 0.420 \\
\hline & Nausea & $10 / 45$ & 22.2 & $3 / 21$ & 14.3 & $7 / 24$ & 29.2 & 0.296 \\
\hline \multirow[t]{2}{*}{ Hochuekkito } & Anorexia & $44 / 90$ & 48.9 & $14 / 36$ & 38.9 & $30 / 54$ & 55.6 & 0.137 \\
\hline & Fatigue & $54 / 96$ & 56.3 & 19/39 & 48.7 & $35 / 57$ & 61.4 & 0.295 \\
\hline \multirow[t]{2}{*}{ Rikkunshito } & Nausea & $36 / 82$ & 43.9 & $9 / 34$ & 26.5 & $27 / 48$ & 56.3 & 0.012 \\
\hline & Anorexia & $46 / 92$ & 50.0 & $18 / 40$ & 45.0 & $28 / 52$ & 53.8 & 0.528 \\
\hline \multirow[t]{2}{*}{ Juzentaihoto } & Fatigue & $50 / 88$ & 56.8 & $17 / 33$ & 51.5 & $33 / 55$ & 60.0 & 0.508 \\
\hline & $\begin{array}{l}\text { AE caused by chemotherapy } \\
\text { or radiotherapy }\end{array}$ & $27 / 58$ & 46.6 & $7 / 22$ & 31.8 & $20 / 36$ & 55.6 & 0.106 \\
\hline \multirow[t]{2}{*}{ Yokukansan } & Delirium & $38 / 60$ & 63.3 & $18 / 26$ & 69.2 & $20 / 34$ & 58.8 & 0.433 \\
\hline & Anxiety & $15 / 50$ & 30.0 & $6 / 23$ & 26.1 & $9 / 27$ & 33.3 & 0.758 \\
\hline \multirow[t]{2}{*}{ Shakuyakukanzoto } & Leg cramps & $79 / 96$ & 82.3 & $36 / 43$ & 83.7 & $43 / 53$ & 81.1 & 0.794 \\
\hline & Abdominal pain & $20 / 57$ & 35.1 & $11 / 25$ & 44.0 & $9 / 32$ & 28.1 & 0.268 \\
\hline \multirow[t]{2}{*}{ Daikenchuto } & lleus & $101 / 128$ & 78.9 & $35 / 48$ & 72.9 & $66 / 80$ & 82.5 & 0.263 \\
\hline & $\begin{array}{l}\text { Opioid-caused constipation } \\
\text { and abdominal pain }\end{array}$ & $62 / 115$ & 53.9 & $22 / 47$ & 46.8 & $40 / 68$ & 58.8 & 0.254 \\
\hline \multirow[t]{2}{*}{ Goshajinkigan } & Numbness of hands and feet & $47 / 107$ & 43.9 & $18 / 39$ & 46.2 & $29 / 68$ & 42.6 & 0.840 \\
\hline & Nocturia & $13 / 60$ & 21.7 & $4 / 26$ & 15.4 & $9 / 34$ & 26.5 & 0.358 \\
\hline
\end{tabular}

Multiple answers allowed, p-value based on Chi-square test. 
Table 7 Open issues about prescribing Kampo medicine $(\mathbf{n}=\mathbf{2 8 5})$

\begin{tabular}{lll}
\hline Issue & frequency & \% \\
\hline The dose and dosage forms need to be better devised for simpler application & 173 & 109 \\
\hline No evidence of efficacy from placebo-controlled studies & 97 & 38.7 \\
\hline Action mechanism of Kampo medicine is not yet elucidated & 90 & 34.0 \\
\hline No opportunity to learn about Kampo medicines & 79 & 31.6 \\
\hline Relatively weak effect & 66 & 27.7 \\
\hline Drug interaction is uncertain & 56 & 23.2 \\
\hline Production of effect is slow & 25 & 19.6 \\
\hline Others & 12 & 8.8 \\
\hline There are no issues & 4.2 \\
\hline
\end{tabular}

Multiple answers allowed.

to combine with other drugs' ( $\mathrm{n}=149,80.5 \%)$. The palliative care specialists tended to place more importance than the non-specialists on 'patient demand' $(\mathrm{p}=0.050)$.

\section{Open issues for prescription}

The questionnaire also asked the physicians to identify any open issues regarding the prescription of Kampo medicines (Table 7$)$, revealing that $60.7 \%(n=173)$ of the physicians were concerned that the dose and dosage forms need to be better devised for simpler administration. Kampo medicines are commonly prepared in granule form or as decoctions, and their administration method is nauseating for some patients. This issue may be related to the observation that "patient demand" was chosen least frequently as the reason for prescription. In the clinical field of palliative care, Kampo medicines are often mixed in a jelly for patients who have dysphagia. For future prescriptions, the administration forms need to be better devised from an adherence perspective. The second most frequently identified issue was the lack of scientific evidence for their efficacy, with $38.2 \%(n=109)$ of the physicians highlighting the absence of evidence from placebo-controlled trails. Watanabe et al. [3] recently reported a summary of 135 peer-reviewed Kampo trials published between 1988 and 2007. According to their report, 106 trials were RCTs, and only 22 were placebo-controlled trials. In two-thirds of the trials, the sample size was less than 100 patients, and only 35 trials were published in English and the rest were in Japanese. Watanabe et al. [3] concluded that the overall quality of the research was low.

\section{Conclusions}

We conducted a nationwide survey of 311 physicians working in palliative care teams at core cancer treatment hospitals and PCUs within medical facilities. Kampo medicines were prescribed by a high proportion $(n=200$, $64.3 \%$ ) of the palliative care physicians and were expected to provide valid means of controlling the cancer patients' symptoms or the adverse side effects of chemotherapy. Palliative care physicians appear to be aware of the effectiveness of Kampo medicines. However, they prescribe Kampo medicines only to a limited extent because of the lack of evidence for their efficacy. Hence, we believe that the collection of more evidence from clinical studies is desirable in Japan.

\section{Abbreviations}

MHLW: Ministry of health labour and welfare; CAM: Complementary and alternative medicine; PCUs: Palliative care units.

\section{Competing interests}

The authors declare that they have no competing interests. The authors were free to interpret the data according to a strict scientific rationale.

\section{Authors' contributions}

YU conceived the study idea and SI and TY contributed to the study design and concept. YU distributed and collected the questionnaires. SI, TY and TM processed and analyzed the data. SI and TM wrote the initial manuscript. All authors interpreted the data and approved the final manuscript.

\section{Acknowledgments}

This work was supported by Grants-in-Aid for the Third-term Comprehensive 10-year Strategy for Cancer Control from the Ministry of Health, Labour and Welfare, Japan and the Foundation for Promotion of Cancer Research in Japan, as well as a Grant-in-Aid for Scientific Research from the Ministry of Education, Culture, Sports Science and Technology of Japan.

\section{Author details}

'Department of Palliative Medicine, The University of Tokyo Hospital, 7-3-1 Hongo, Bunkyo-ku, Tokyo 113-0033, Japan. '2Division of Biostatistics, Tohoku University Graduate School of Medicine, 1-1 Seiryo-machi, Aoba-ku, Sendai, Miyagi 980-8574, Japan. ${ }^{3}$ Interfaculty Initiative in Information Studies, The University of Tokyo, 7-3-1 Hongo, Bunkyo-ku, Tokyo 113-0033, Japan. ${ }^{4}$ Division of Cancer Pathophysiology, National Cancer Center Research Institute, 5-1-1 Tsukiji, Chuo-ku, Tokyo 104-0045, Japan. ${ }^{5}$ Department of Psychosomatic Internal Medicine, Kagoshima University Graduate School of Medical and Dental Sciences, 8-35-1 Sakuragaoka, Kagoshima 890-8520, Japan.

Received: 15 May 2012 Accepted: 13 November 2012 Published: 20 November 2012

\section{References}

1. Yu F, Takahashi T, Moriya J, Kawaura K, Yamakawa J, Kusaka K, Itoh T, Morimoto S, Yamaguchi N, Kanda T: Traditional Chinese medicine and Kampo: a review from the distant past for the future. J Int Med Res 2006 34:231-239

2. Terasawa K: Evidence-based reconstruction of Kampo medicine: Part I -Is Kampo CAM? Evid Based Complement Altern Med 2004, 1:11-16. 
3. Watanabe K, Matsuura K, Gao P, Hottenbacher L, Tokunaga H, Nishimura K, Imazu Y, Reissenweber H, Witt CM: Traditional Japanese Kampo medicine: Clinical research between modernity and traditional medicine-the state of research and methodological suggestions for the future. Evid Based Complement Altern Med 2011, 2011:1-19.

4. Nishimura K, Plotnikoff GA, Watanabe K: Kampo medicine as an integrative medicine in Japan. Jpn Med Assoc J 2009, 52(3):147-149.

5. Watanabe K: General Practitioner Should Use Kampo Medicine. http://mric. tanaka.md/2008/10/22/_vol_148.html.

6. Yamashita $\mathrm{H}$, Tsukayama $\mathrm{H}$, Sugishita C: Popularity of complementary and alternative medicine in Japan: a telephone survey. Complement Ther Med 2002, 10(2):84-93.

7. Hori S, Mihaylov I, Vasconcelos JC, McCoubrie M: Patterns of complementary and alternative medicine use amongst outpatients in Tokyo. Japan. BMC Complement Altern Med 2008, 8:14

8. Hyodo I, Amano N, Eguchi K, Narabayashi M, Imanishi J, Hirai M, Nakano T, Takashima S: Nationwide survey on complementary and alternative medicine in cancer patients in Japan. J Clin Oncol 2005, 23(12):2645-2654.

9. Harris PE, Cooper KL, Relton C, Thomas KJ: Prevalence of complementary and alternative medicine (CAM) use by the general population: a systematic review and update. Int J Clin Pract 2012, 66:915-916.

10. Molassiotis A, Fernadez-Ortega P, Pud D, Ozden G, Scott JA, Panteli V, Margulies A, Browall M, Magri M, Selvekerova S, Madsen E, Milovics L, Bruyns I, Gudmundsdottir G, Hummerston S, Ahmad AMA, Platin N, Kearney $\mathrm{N}$, Patiraki E: Use of complementary and alternative medicine in cancer patients: a European survey. Ann Oncol 2005, 16:655-663.

11. Arai I: The current situation of the Japanese medical plants industry and its significance for the pharmaceutical industry. http://hdais.coa.gov.tw/ htmlarea_file/web_articles/hdais/1354/980108_1.pdf.

12. Miyoshi A, Yachi A, Masamune $O$, Ishikawa M, Fukutomi $H$, Niwa $H$, Matsuo Y, Mori H, Harasawa S: Clinical evaluation of Rikkunshito (TJ-43 TSUMURA $\&$ Co.) for indeterminate digestive complaints including chronic gastritis, etc.. Multi-institutional study with reference drug of Cisapride. Prog Med 1991, 11:1605-3. in Japanese.

13. Tatsuta M, lishi H: Effect of treatment with liu-jun-zi-tang (TJ-43) on gastric emptying and gastrointestinal symptoms in dyspeptic patients. Aliment Pharmacol Ther 1993, 7:459-462.

14. Harasawa S, Miyoshi A, Miwa T, Masamune O, Matsuo Y, Nakazawa S, Suyama T, Hayakawa A, Nakajima M: Clinical test of ataxia-type upper abdominal disorder (dysmotility-like dyspepsia) after multiple facilities cooperated marketing of Kampo medicine TJ-43 Rikkunnshitou. Examination by double blind experiment group comparison. J Clin Exp Med (IGAKKU NO AYUMI) 1998, 187:207-229. in Japanese.

15. Gochi A, Hirose S, Sato K, Hiramatsu S, Asakura A, Tokuoka H, Matsuno T, Kamikawa Y, Orita K: The Effect of Hange-shashin-to and Rikkunshi-to against the digestive symptoms after gastrectomy. Jpn J Gastroenterol Surg 1995, 28:961-965. in Japanese.

16. Nakajima O, En S: Comparative analysis of the Kampo medicine Rikkunshito's clinical efficacy on patients with functional dyspepsia. Jpn J Med Pharmacol Sci 2008, 59:235-240. in Japanese.

17. Endo G: Analysis of the Rikkunshito efficacy on patients with functional dyspepsia. Jpn J Med Pharmacol Sci 2008, 60:547-552. in Japanese.

18. Oka T, Tamagawa Y, Hayashida S, Kaneda Y, Kodama N, Tsuji S: Rikkunshi-to attenuates adverse gastrointestinal symptoms induced by fluvoxamine. Biopsychosoc Med 2007, 1:21.

19. Okabe T, Tsutani K: Evidence Reports of Kampo Treatment (EKAT) Appendix 2011. http://www.jsom.or.jp/medical/ebm/ere/pdf/EKATE_Appendix_2011. pdf.

20. Uezono $Y$, Miyano $K$, Sudo $Y$, Suzuki M, Shiraishi S, Terawaki K: A review of traditional japanese medicines and their potential mechanism of action. Curr Pharm Des 2012, 18:1-15. In Press.

21. Cancer Control Information Center, National Cancer Center: Cancer Information Service. http://ganjoho.jp/public/index.html.

22. Kautio AL, Haanpää M, Kautiainen H, Kalso E, Saarto T: Burden of chemotherapy-induced neuropathy-a cross-sectional study. Support Care Cancer 2010, 19:1991-1996.

23. Cella D, Lai JS, Chang CH, Peterman A, Slavin M: Fatigue in cancer patients compared with fatigue in the general United States population. Cancer 2002, 94(2):528-538.
24. Breitbart W, Bruera E, Chochinov H, Lynch M: Neuropsychiatric syndromes and psychological symptoms in patients with advanced cancer. J Pain Symptom Manage 1995, 10:131-141.

25. Massie MJ, Holland JC, Glass E: Delirium in terminally ill cancer patients. Am J Psychiatry 1983, 140:1048-1050.

26. Lawlor PG, Gagnon B, Mancini IL, Pereira JL, Hanson J, Suarez-Almazor ME, Bruera ED: Occurrence, causes and outcomes of delirium in patients with advanced cancer. Arch Intern Med 2000, 160:786-794.

27. Ravasco P: Aspects of taste and compliance in patients with cancer. Eur J Oncol Nurs 2005, 9:84-91.

28. Tsumura \& Co: Product Information. http://www.tsumura.co.jp/password/ m_square/products/ichiran/ (in Japanese).

29. Arita M: Crude Drug Lsit. http://metabolomics.jp/wiki/Persist:CrudeDrugList

30. The Japanese pharmacopeia fifteenth edition. http://jpdb.nihs.go.jp/.jp15/ (in Japanese).

31. A tolerability and efficacy study of TU-100 for the treatment of postoperative ileus. http://clinicaltrials.gov/ct2/show/NCT00266461?term=Daikenchuto+\% 28TU-100\%29\&rank=3.

doi:10.1186/1472-6882-12-222

Cite this article as: Iwase et al:: The clinical use of Kampo medicines (traditional Japanese herbal treatments) for controlling cancer patients' symptoms in Japan: a national cross-sectional survey. BMC Complementary and Alternative Medicine 2012 12:222.

\section{Submit your next manuscript to BioMed Central and take full advantage of:}

- Convenient online submission

- Thorough peer review

- No space constraints or color figure charges

- Immediate publication on acceptance

- Inclusion in PubMed, CAS, Scopus and Google Scholar

- Research which is freely available for redistribution

Submit your manuscript at www.biomedcentral.com/submit
C BioMed Central 